\title{
Arachnoiditis Following Caudal Epidural Injections for the Lumbo-Sacral Radicular Pain
}

\author{
Shashi Kumar Nanjayan, Girish Nanjunda Swamy, Sachin Yallappa, Rajendra Bommireddy \\ Department of Trauma and Orthopaedics, Royal Derby Hospital, Derby, UK
}

\begin{abstract}
Caudal epidural steroid injection is a very common intervention in treatment of low back pain and sciatica symptoms. Although extensively used, it is not devoid of complications. A few reports of chemical and infective arachnoiditis exist following lumbar epidural anaesthesia, but none following a caudal epidural steroid injection. We report a case of arachnoiditis following caudal epidural steroid injections for lumbar radiculopathy. The patient presented with contralateral sciatica, worsening low back pain and urinary retention few days following the injection, followed by worsening motor functions in L4/L5/S1 myotomes with resultant dense foot drop. Gadolinium-enhanced magnetic resonance imaging suggested infective arachnoiditis with diffuse enhancement and clumping of the nerve roots within the lumbar and sacral thecal sac. As the number of injections in the management of back pain and lumbo-sacral radicular pain is increasing annually, it is imperative to have a thorough understanding of this potentially dangerous complication and educate the patients appropriately.
\end{abstract}

Keywords: Caudal; Epidural; Low back pain; Sciatica; Arachnoiditis

\section{Introduction}

Chronic low back pain is a major health condition. Caudal epidural steroid injections are one of the commonly used interventions used in managing the chronic low back pain and lower extremity pain [1-3]. Caudal approach to the epidural space was first reported more than a century ago [1]. Herniated nucleus pulposus with or without nerve-root irritation are usually good indications for the caudal epidural injections and usually get the most beneficial effects $[1,4]$.

Caudal epidural injections are not devoid of complications and disadvantages [1]. Epidural steroid injections have been reported to meet with few complications. The most common complication is a transient headache with or without identifiable dural puncture.
Transient complications have also been reported during the fluoroscopically-guided caudal epidural injections, including insomnia, transient non-positional headaches, increased back pain, facial flushing, vasovagal reactions, nausea and increased leg pain [5].

There are very few published reports of arachnoiditis following epidural steroid injections in the literature and none following a caudal epidural injection. We report a case of arachnoiditis after a caudal epidural injection, which was given for lumbosacral radicular pain.

\section{Case Report}

A 58-year-old male patient was treated with a course of caudal epidural injections for worsened left-sided sciatic/ claudication pain secondary to the lumbar disc prolapse

Received May 29, 2012; Revised Sep 27, 2012; Accepted Sep 27, 2012

Corresponding author: Girish Nanjunda Swamy

Department of Trauma and Orthopaedics, Royal Derby Hospital,

Uttoxeter Road, Derby, DE22 3NE, UK

Tel: +44-7792034317, Fax: +44-1332 254944, E-mail: girishjam@yahoo.com 
at L3/L4 level (Figs. 1, 2). Patient had no constitutional symptoms or red-flag signs.

Pre-injection, the clinical examination revealed a slight altered sensation in the left L4 dermatome with the normal motor examination. The right lower limb neurology was normal. Routine haematological investigations were normal and negative for infections. His medical history included transient ischaemic attacks, ischaemic heart disease, and hypertension.

The left L4 nerve root block offered 2 weeks of painrelief, before the patient experienced a recurrence of the symptoms. The decision to treat with a course of the caudal epidural injections was made. His first caudal epidural injection was uneventful.

Four days following the second caudal epidural injection, the patient presented to the emergency department with a new onset of contralateral (right-sided) sciatica, which had worsened over the last 3 days. The patient's back pain had worsened, and he noticed difficulties in passing urine. He was afebrile and denied any chills, rigors or neck stiffness.

The examination revealed decreased sensations over the left L4, L5, and S1 with Medical Research Council (MRC) grade 3 power. He had absent bilateral knee and ankle reflexes with normal plantar reflexes. Bilateral straight leg rising was positive for sciatic irritation/stretch at 40 degrees.

The repeat magnetic resonance imaging (MRI) suggested a resolved $\mathrm{L} 3, \mathrm{~L} 4$ disc protrusion with no new

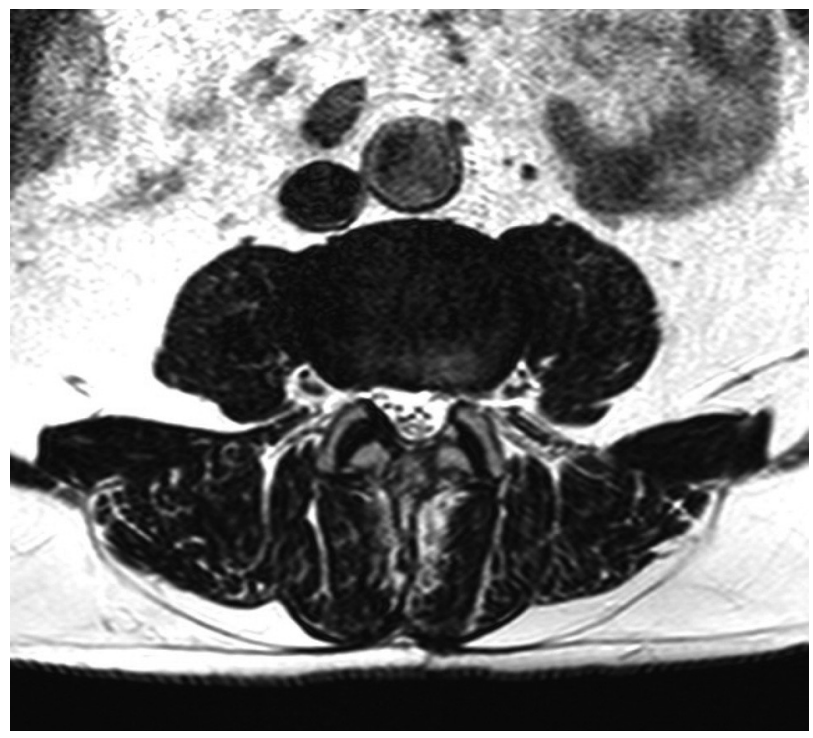

Fig. 1. Axial section at L3/L4 before the caudal epidural injection. findings. The patient subsequently was noted to have a raised temperature of more than 37.8 degree, shooting pain in bilateral legs with associated paraesthesia in both feet. His blood investigations revealed raised inflammatory markers (C-reactive protein [CRP], 277; white cell count [WCC], $12.81 \times 10^{9}$ with neutrophilia).

The patient was started on empirical IV flucloxacillin after three random blood cultures taken during the febrile episodes. These cultures were subsequently found negative for bacterial growth. Multiple attempts at lumbar puncture to obtain the sample of cerebrospinal fluid (CSF; for evaluation) were very difficult and unsuccessful. Subsequently the patient deteriorated with worsening signs and symptoms of sepsis, which warranted a change in empirical IV flucloxacillin to IV vancomycin as per the microbiologist advice. Any further attempt to obtain CSF sample was abandoned at this stage. Following day, the patient developed a full loss of power in his left L4, L5, and S1 nerve roots, leading to full-blown left foot drop.

The repeat MRI at this stage showed diffuse enhancement of the nerve roots within the lumbar and sacral thecal sac which continued upwards. Meningeal enhancement of conus and lower cord was also noted along with 'clumping' of the nerve roots, which suggested infective arachnoiditis (Fig. 3). The patient also had a nerve conduction study, which showed marked denervation changes in the left L5-S1 distribution, indicating radiculopathy.

The patient gradually improved after 2 weeks of intra-

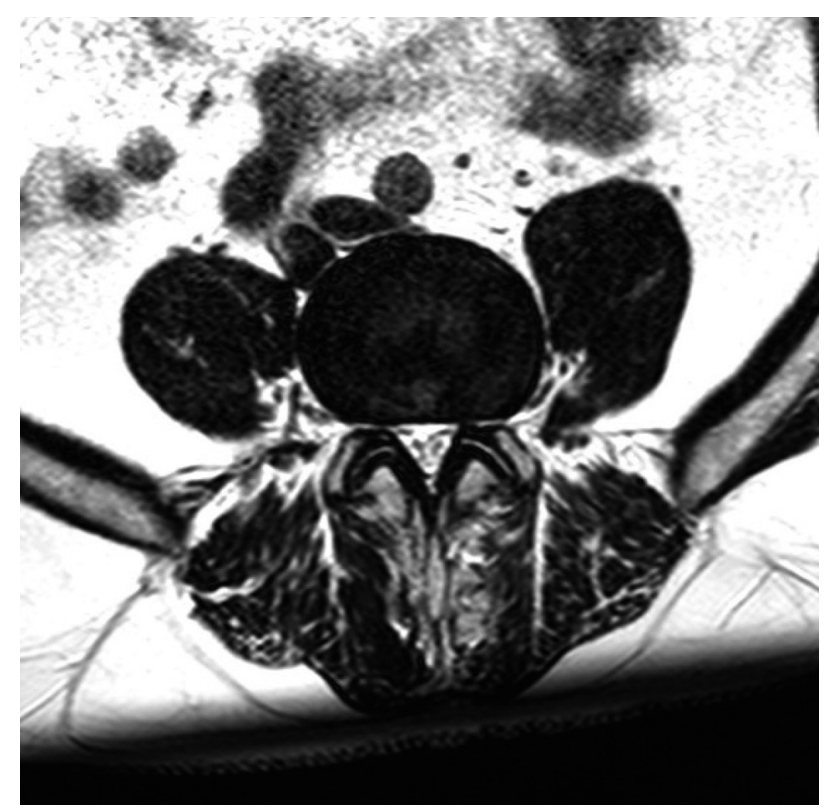

Fig. 2. Axial section of $L 4 / L 5$ before the epidural steroid injection. 
venous antibiotics (vancomycin), and the inflammatory markers (CRP, WCC, and erythrocyte sedimentation rate) returned to the normal limits. He made a slow recovery, but the left-sided foot drop persisted. He was discharged after being administered IV antibiotics for fourweek duration. At the 6-month follow-up, the patient had no clinical signs of infection and was generally well with the normal inflammatory markers in the blood test. His left foot drop still persists.

\section{Discussion}

At our institute, we use a mixture of $7 \mathrm{~mL}$ of $1 \%$ lidocaine, $80 \mathrm{mg}$ of triamcinolone and $7 \mathrm{~mL}$ of normal saline in the caudal epidural injections, with the main indication being radicular lumbosacral pain.

Although caudal epidural injections are safe, they are not devoid of complications. At our institute, we perform more than 1,500 caudal epidural steroid injections per year, and the rate of infections are less than $0.1 \%$. If any suggestions of infection are seen, aggressive approaches in the investigation and management should be undertaken at the first opportunity. If delayed, the consequences can be devastating.

Meningitis and epidural abscess have been reported fol- lowing the epidural injections [6]. Arachnoiditis is very rare, especially following a caudal epidural injection. Adhesive arachnoiditis may result from the solvent of deposteroid polyethylene glycol [1]. Infections associated with the contaminated drugs prepared at the compounding pharmacies have been reported, following the epidural injections in the out-patient pain management clinics [7].

The return of these patients to the emergency department or the back pain/spinal clinic has to be dealt with a fresh eye and approach. In the setting of the febrile episodes or new symptoms, the infective complications of the caudal epidural injection have to be borne in mind by the treating physicians. A delay or failure to recognise such presentation could lead to devastating outcomes. The radiological picture in such a setting can be very subtle, and it can easily escape an inexperienced eye. With this report, we would like to raise awareness of arachnoiditis following caudal epidural injections.

The radiological picture of arachnoiditis includes abnormal conglomeration of nerve roots or clumping, which has been seen in our patients [8].

Our patient had symptoms and signs of meningeal and nerve root irritation four-days following the second epidural injection. Despite the multiple attempts by a very experienced senior consultant anaesthetist, the CSF sam-
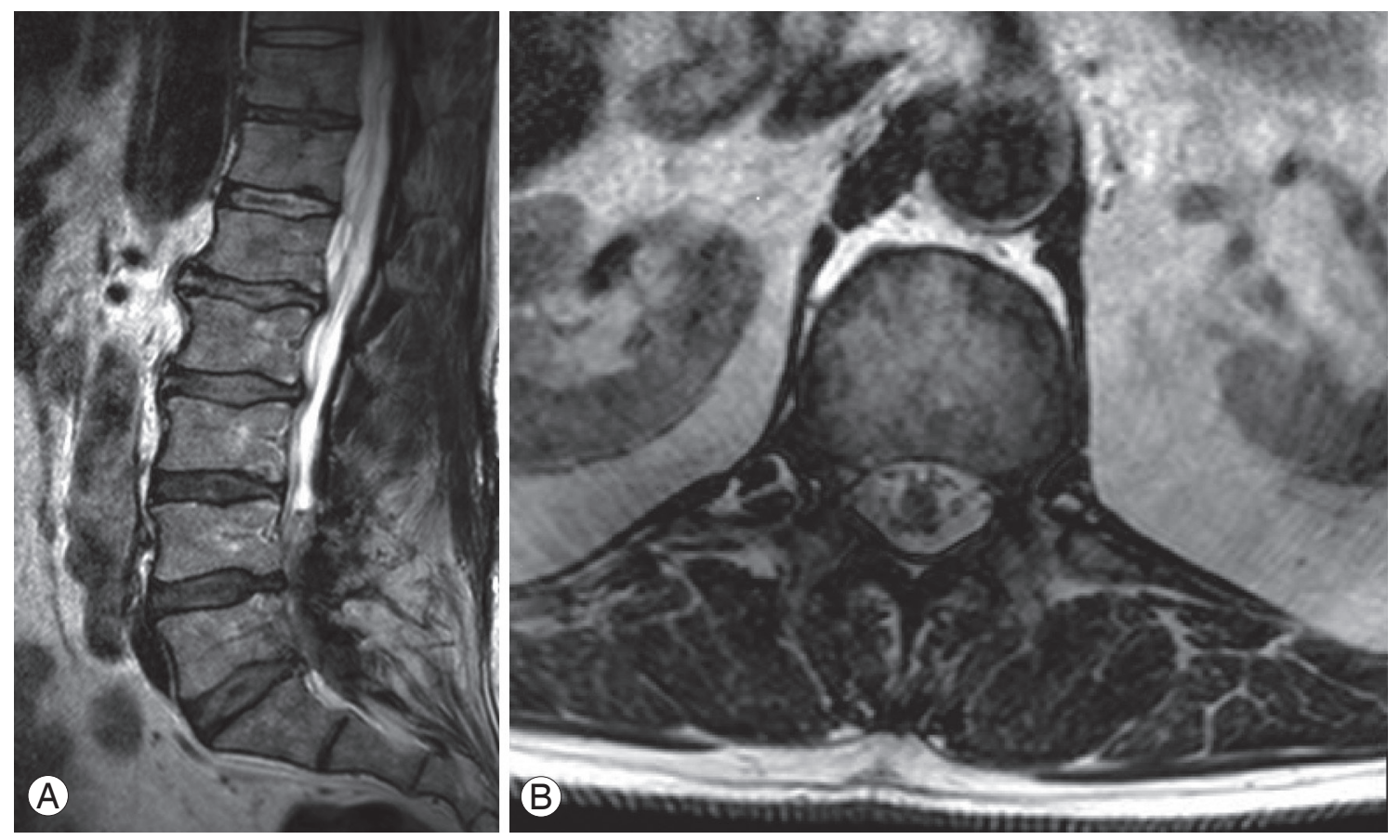

Fig. 3. (A) Sagittal section and (B) Axial section at L1. Axial section at L1 with Gadolinium enhancement. More pronounced meningeal thickening with clumping of the lower lumbar nerve roots consistent with arachnoiditis. 
ple could not be obtained. The difficulties in obtaining the CSF sample was a shortcoming in our patient-management, but further attempts for the lumbar puncture was abandoned in the best interest of the patient. Chemical arachnoiditis from the preservatives in triamcinolone was considered unlikely given the significant raise in the inflammatory markers in the blood.

As the number of injections and interventions in the management of the back pain and lumbosacral radicular pain is increasing annually, the pain physicians and doctors who are involved in the care of these patients need to be aware of complications such as arachnoiditis, which should be considered in the differential diagnosis.

\section{Conflict of Interest}

No potential conflict of interest relevant to this article was reported.

\section{References}

1. Ogoke BA. Caudal epidural steroid injections. Pain Physician 2000;3:305-12.
2. Conn A, Buenaventura RM, Datta S, Abdi S, Diwan S. Systematic review of caudal epidural injections in the management of chronic low back pain. Pain Physician 2009;12:109-35.

3. White AH, Derby R, Wynne G. Epidural injections for the diagnosis and treatment of low-back pain. Spine (Phila Pa 1976) 1980;5:78-86.

4. Spaccarelli KC. Lumbar and caudal epidural corticosteroid injections. Mayo Clin Proc 1996;71:169-78.

5. Botwin KP, Gruber RD, Bouchlas CG, et al. Complications of fluoroscopically guided caudal epidural injections. Am J Phys Med Rehabil 2001;80:416-24.

6. Hooten WM, Kinney MO, Huntoon MA. Epidural abscess and meningitis after epidural corticosteroid injection. Mayo Clin Proc 2004;79:682-6.

7. From the Centers for Disease Control and Prevention. Exophiala infection from contaminated injectable steroids prepared by a compounding pharmacy: United States, July-November 2002. JAMA 2003;289:291-3.

8. Ross JS, Masaryk TJ, Modic MT, et al. MR imaging of lumbar arachnoiditis. AJR Am J Roentgenol 1987;149:1025-32. 